\title{
INFECTIOUS BRONCHITIS CORONAVIRUS INDUCES CELL-CYCLE PERTURBATIONS
}

\author{
Brian K. Dove, Katrina Bicknell, Gavin Brooks, Sally Harrison, \\ and Julian A. Hiscox*
}

\section{INTRODUCTION}

The term 'cell cycle' is a generic description comprising the various stages that actively replicating cells undergo to proliferate. The cell cycle of replicating mammalian cells can be divided into five distinct phases ${ }^{1}$ : gap 1 and gap $2\left(\mathrm{G}_{1}\right.$ and $\left.\mathrm{G}_{2}\right)$ where cells undergo RNA and protein synthesis, synthesis $(\mathrm{S})$ phase where cellular DNA replication occurs, and mitosis (M) phase followed by cytokinesis (cell division) (Fig. 1). Cells not undergoing replication and within a quiescent state are described as being in $\mathrm{G}_{0}$ phase.

Viruses from a diverse range of families have been shown to be able to perturb the cell cycle of infected cells, ${ }^{2-6}$ including the coronavirus mouse hepatitis virus, which induces a $G_{1}$ arrest. $^{7}$ Although the primary site of coronavirus replication is the cytoplasm, localization and interactions of coronavirus proteins with nuclear and subnuclear structures and proteins have been reported..$^{8-11}$ Therefore, we hypothesize that avian infectious bronchitis virus (IBV), a group 3 member of the coronavirus family, would induce cell-cycle perturbations as a consequence of virus infection.

To investigate this, we utilized dual-label flow cytometric analysis to accurately gate cells in the $G_{0} / G_{1}, S$ and $G_{2} / M$ phases of the cell cycle. ${ }^{12,13}$ Prototypic single-color flow cytometric analysis of cycling cells uses propidium iodide (PI) to stain the total cellular DNA content of individual cells. This allows measurement of the percentage of cells in the $\mathrm{G}_{0} / \mathrm{G}_{1}$ (2N DNA content), $\mathrm{G}_{2} / \mathrm{M}$ (4N DNA content), and $\mathrm{S}$ (intermediate DNA content) phases within a cell population. However, application of a dual-label approach, by the addition of thymidine analogue bromodeoxyuridine (BrdU) incorporated into DNA during cellular DNA synthesis allows increased discrimination and measurement of cell populations into the $\mathrm{G}_{0} / \mathrm{G}_{1}, \mathrm{~S}$, and $\mathrm{G}_{2} / \mathrm{M}$ phases.

\footnotetext{
* Brian K. Dove, Sally Harrison, Julian A. Hiscox, University of Leeds, Leeds LS2 9JT United Kingdom. Katernia Bicknell, Gavin Brooks, University of Reading, Reading RG6 6AJ, United Kingdom.
} 


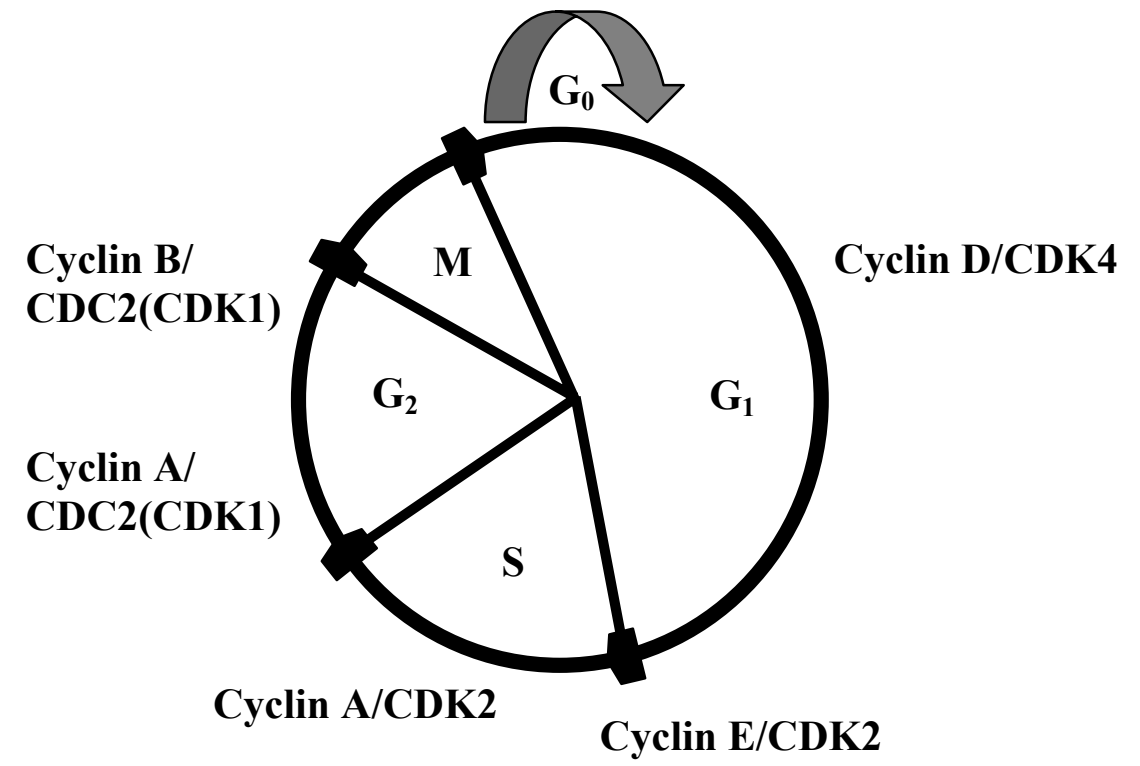

Figure 1. The five cellular phases that compr. the mammalian cell cycle. Positive control is regulated by the formation of specific cyclin/cyclin-dependent kinase (CDK) complexes, required at certain stages of the cell cycle for progression to occur.

\section{MATERIALS AND METHODS}

\subsection{IBV Infections}

Actively replicating asynchronous Vero cells (an African green monkey kidneyderived kidney cell line) were infected with either IBV B-US strain at an MOI of 1, ultraviolet (UV) inactivated IBV B-US, or mock infected. Cells were analyzed at the appropriate time postinfection (pi) by dual-label flow cytometric analysis.

\subsection{Dual-Labeled Flow Cytometric Analysis}

Two-color flow cytometric analysis was used to accurately determine the cell-cycle profile of both mock and infected cell populations. BrdU was added to cell medium within each flask 30 minutes prior to fixing the cells. BrdU-labeled DNA was detected by addition of mouse-anti-BrdU antibody followed by anti-mouse FITC antibody. The PI stain was then applied and the cell populations were analyzed for PI staining and BrdU incorporation using a FACS Calibur analyzer (Becton Dickinson) and the percentage of cells in the $G_{0} / G_{1}, S$, or $G_{2} / M$ phases in each sample gated using CellQuest software (Becton Dickinson).

\subsection{Cyclin-Dependent Kinase Assay}

Cyclin-dependent kinase 2 (CDK2) and CDC2 (CDK1) associated complexes were immunoprecipitated from mock and IBV-infected Vero cells at $0,8,16$, and $24 \mathrm{hr}$ 
post-infection. Complexes were immunoprecipitated from total protein extracts using anti-CDK2 polyclonal antibody or anti-cyclin B1 monoclonal antibody, respectively. Associated kinase activities were measured using histone $\mathrm{H} 1$ as substrate. Phosphorylation of histone H1 was analyzed using a Molecular Dynamics PhosphorImager SI and quantified using the ImageQuant software package (Molecular Dynamics).

\subsection{Western Blot Analysis of Cellular Proteins}

Total cellular protein, extracted from mock and IBV-infected Vero cells at various times pi, was separated on a $10 \%$ Novex Bis-Tris polyacryamide pre-cast gel in MES SDS running buffer (Invitrogen). Western blotting was performed using ECL (Amersham/Pharmacia) as described in the manufacturer's instructions.

\section{RESULTS AND DISCUSSION}

To determine any IBV-infection induced cell-cycle perturbations, asynchronously replicating Vero cells were infected with both IBV and UV-inactivated IBV as well as mock-infected. Dual-label flow cytometric analysis determined that at $24 \mathrm{hr}$ pi, there was a significant increase $(p<0.001, \mathrm{n}-3)$ in the number of cells in the $\mathrm{G}_{2} / \mathrm{M}$ phase of the cell cycle compared with both mock-infected cells and cells infected with UV-inactivated virus. There was also a significant decrease in the number of cells in the $G_{0} / G_{1}$ phase of the cell cycle in infected cells compared with the controls $(p<0.001, \mathrm{n}-3)$. While not discussed in this chapter, a similar $\mathrm{G}_{2} / \mathrm{M}$ phase arrest was also observed in IBV-infected BHK cells, another IBV-permissible cell line.

Studies also focused on identifying any IBV-induced perturbations and interactions with specific cell-cycle factors. Both CDK2 and CDC2 kinase assays were performed to determine any alteration in the level of kinase activity of cyclinB/CDC2 complexes and of both cyclin A/CDK2 and E/CDK2 complexes in IBV infected cells. Figure 3 shows that while kinase activity fluctuated over time in both mock and infected cells, at $24 \mathrm{hr}$ pi CDK2 activity was reduced in IBV-infected Vero cells compared with mock-infected. Therefore, infection with IBV resulted in a reduction of either/or cyclin E/CDK2 and cyclin A/CDK2 activity. This reduction in activity in infected cells may be due to a reduced number of cells cycling through the cell cycle as a consequence of the virus-induced block. Western blot analysis was also performed on a variety of both positive and negative cellular cell

Table 1. IBV-infected Vero cell cycle profile.

\begin{tabular}{lccc}
\hline \multirow{2}{*}{ Infectious state } & \multicolumn{3}{c}{ Cell-cycle phase percentages } \\
\cline { 2 - 4 } & $\mathrm{G}_{0} / \mathrm{G}_{1}$ & $\mathrm{~S}$ & $\mathrm{G}_{2} / \mathrm{M}$ \\
\hline Mock infected & $69.9+/-0.7$ & $21.3+/-1.0$ & $8.8+/-1.7$ \\
U.V. inactive IBV & $69.2+/-1.0$ & $22.7+/-0.6$ & $8.0+/-0.5$ \\
IBV infected & $59.6+/-1.2^{*}$ & $17.8+/-2.1$ & $22.3+/-1.8^{*}$ \\
\hline
\end{tabular}

Specific cell-cycle stage percentages were calculated from BrdU/PI dual-label dot blots (Figure 2 ) $+/$ - standard deviation. *, significantly different from corresponding mock infected and UV-inactivated cell controls $(p<$ $0.001 ; \mathrm{n}-3)$. 
cycle regulatory factors. Analysis of mock-infected and IBV-infected Vero cell lysates for cyclins D1, D2, A, E, and B determined that at 16 and $24 \mathrm{hr}$ a 10-and 16-fold reduction, respectively, of both cyclin D1 and D2 expression in IBV-infected cells, compared with mock infected cells, was detectable (data not shown). A reduction in cyclin D expression was also reported by Chen et al. ${ }^{7}$ Analysis of cyclin A, E, and B expression, while undergoing slight perturbations, demonstrated no significant change in the level of expression between mock-infected and IBV-infected Vero cells. Western blot analysis of the negative cellular regulatory protein $\mathrm{p} 21$ and tumor suppressor protein $\mathrm{p} 53$ also demonstrated no significant alteration in the levels of p21 and p53 expressed between mock and infected cells (data not shown).

Our data indicate that IBV infection induces a $\mathrm{G}_{2} / \mathrm{M}$ phase arrest or delay within infected cells. While Chen et al. ${ }^{7}$ reported that MHV induced a $G_{0} / G_{1}$ phase arrest in cells, the difference in cell-cycle arrest state between IBV and MHV could simply be due to the inherent differences between the viruses or the type of cell-cycle analysis used. Currently, work is focused on the development of a model to explain how IBV induces a $\mathrm{G}_{2} / \mathrm{M}$ phase arrest and to determine what physiological advantage a $G_{2} / M$ phase arrest confers to IBV infection. Preliminary work indicates both an increase of viral protein production and progeny virus output in cells $G_{2} / M$ synchronized compared with $G_{0} / G_{1}$ synchronized cells and asynchronously replicating cells (data not shown). Therefore, identification of the mechanism or mechanisms IBV manipulates to induce a cell-cycle arrest could lead to further insight into the mechanism of IBV replication.
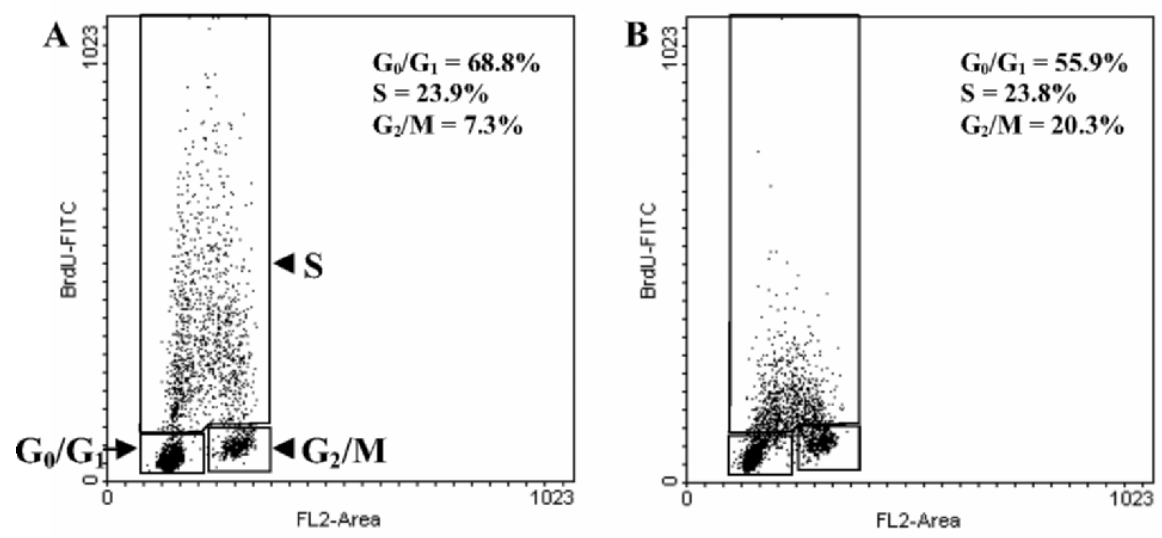

Figure 2. Representative BrdU-labeled (BrdU-FITC) / PI-stained (FL2-area) dot blot cell-cycle profiles of mock-infected (A) and IBV-infected (B) Vero cells at $20 \mathrm{hr}$ pi. Utilizing a dual-label approach allows accurate gating of a cell population into the $\mathrm{G}_{0} / \mathrm{G}_{1}, \mathrm{~S}$, and $\mathrm{G}_{2} / \mathrm{M}$ phases of the cell cycle. 

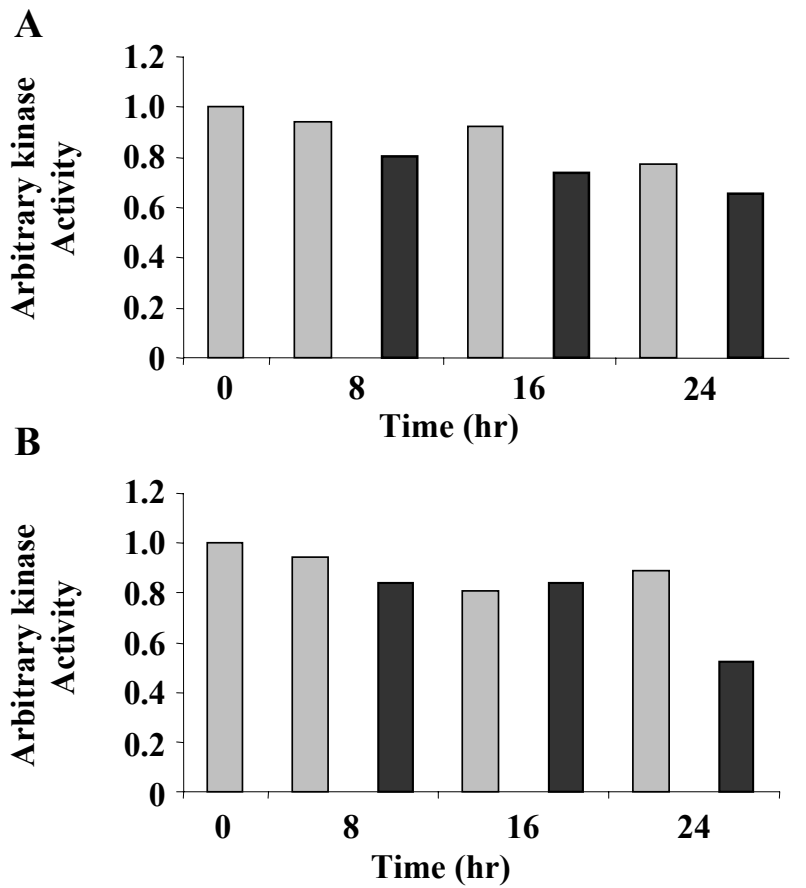

Figure 3. The level of histone $\mathrm{H} 1$ kinase activity from CDC2- (A) and CDK2- (B) associated complexes immunoprecipitated from total cellular protein extracted from mock-infected and IBV-infected Vero cells at 0 , 8,16 , and $24 \mathrm{hr}$ postinfection was quantified to calculate arbitrary kinase activity in mock-infected (gray) and IBV-infected cells (black). IBV infection results in a reduction of CDK2 kinase activity.

\section{ACKNOWLEDGMENTS}

This work was funded by the BBSRC (grant number BBS/B/03416) to J.A.H. and G.B. We would like to acknowledge the help and expertise of Graham Botley of the University of Leeds and Carmen Coxon and Jane Harper of the University of Reading with the flow cytometry. The flow cytometer at the University of Leeds was provided by a grant from Yorkshire Cancer Research.

\section{REFERENCES}

1. J. V. Harper and G. Brooks, The mammalian cell cycle: an overview, Meth. Mol. Biol. 296, 113-153 (2005)

2. K. L. Tyler, P. Clarke, R. L. DeBiasi, D. Kominsky, and G. J. Poggioli, Reoviruses and the host cell, Trends Microbiol. 9, 560-564 (2001).

3. A. Op De Beeck and P. Caillet-Fauquet, Viruses and the cell cycle, Prog. Cell Cycle Res. 3, 1-19 (1997).

4. E. K. Flemington, Herpesvirus lytic replication and the cell cycle: arresting new developments, J. Virol. 75 , 4475-4481 (2001).

5. D. Naniche, S. I. Reed, and M. B. Oldstone, Cell cycle arrest during measles virus infection: a G0-like block leads to suppression of retinoblastoma protein expression, J. Virol. 73, 1894-1901 (1999).

6. B. Groschel and F. Bushman, Cell cycle arrest in G2/M promotes early steps of infection by human immunodeficiency virus, J. Virol. 79, 5695-5704 (2005). 
7. C. J. Chen and S. Makino, Murine coronavirus replication induces cell cycle arrest in G0/G1 phase, J. Virol. 78, 5658-5669 (2004).

8. J. A. Hiscox, T. Wurm, L. Wilson, P. Britton, D. Cavanagh, and G. Brooks, The coronavirus infectious bronchitis virus nucleoprotein localizes to the nucleolus, J. Virol. 75, 506-512 (2001).

9. T. Wurm, H. Chen, T. Hodgson, P. Britton, G. Brooks, and J. A. Hiscox, Localization to the nucleolus is a common feature of coronavirus nucleoproteins, and the protein may disrupt host cell division, J. Virol. 75, 9345-9356 (2001).

10. H. Chen, T. Wurm, P. Britton, G. Brooks, and J. A. Hiscox, Interaction of the coronavirus nucleoprotein with nucleolar antigens and the host cell, J. Virol. 76, 5233-5250 (2002).

11. X. Yuan, Z. Yao, Y. Shan, B. Chen, Z. Yang, J. Wu, Z. Zhao, J. Chen, and Y. Cong, Nucleolar localization of non-structural protein $3 \mathrm{~b}$, a protein specifically encoded by the severe acute respiratory syndrome coronavirus, Virus Res. (2005).

12. F. Dolbeare, H. Gratzner, M. G. Pallavicini, and J. W. Gray, Flow cytometric measurement of total DNA content and incorporated bromodeoxyuridine, Proc. Natl. Acad. Sci. USA 80, 5573-5577 (1983).

13. R. Nunez, DNA measurement and cell cycle analysis by flow cytometry, Curr. Issues Mol. Biol. 3, 67-70 (2001). 